\title{
Brain mechanisms of flavor learning
}

\section{Takashi Yamamoto * and Kayoko Ueji}

Department of Health and Nutrition, Faculty of Health Science, Kio University, Nara, Japan

\section{Edited by:}

Milagros Gallo, University of Granada, Spain

\section{Reviewed by:}

Federico Bermudez-Rattoni,

Universidad Nacional Autónoma de

México, Mexico

Milagros Gallo, University of Granada, Spain

*Correspondence:

Takashi Yamamoto, Department of Health and Nutrition, Faculty of

Health Science, Kio University, 4-2-4

Umami-naka, Koryo-cho, Nara

635-0832, Japan.

e-mail: ta.yamamoto@kio.ac.jp
Once the flavor of the ingested food (conditioned stimulus, CS) is associated with a preferable (e.g., good taste or nutritive satisfaction) or aversive (e.g., malaise with displeasure) signal (unconditioned stimulus, US), animals react to its subsequent exposure by increasing or decreasing ingestion to the food. These two types of association learning (preference learning vs. aversion learning) are known as classical conditioned reactions which are basic learning and memory phenomena, leading selection of food and proper food intake. Since the perception of flavor is generated by interaction of taste and odor during food intake, taste and/or odor are mainly associated with bodily signals in the flavor learning. After briefly reviewing flavor learning in general, brain mechanisms of conditioned taste aversion is described in more detail. The CS-US association leading to long-term potentiation in the amygdala, especially in its basolateral nucleus, is the basis of establishment of conditioned taste aversion. The novelty of the CS detected by the cortical gustatory area may be supportive in CS-US association. After the association, CS input is conveyed through the amygdala to different brain regions including the hippocampus for contextual fear formation, to the supramammillary and thalamic paraventricular nuclei for stressful anxiety or memory dependent fearful or stressful emotion, to the reward system to induce aversive expression to the CS, or hedonic shift from positive to negative, and to the CS-responsive neurons in the gustatory system to enhance the responsiveness to facilitate to detect the harmful stimulus.

Keywords: conditioning, taste, odor, aversion, preference, brain
Food and fluid intake is one of the most essential behaviors since animals require adequate nutrients and reject toxins for their survival. Although energy homeostasis is a basis of regulating food and fluid intake, actual ingestive behavior in animals including humans is controlled by innate and learned flavor preference and/or aversion. Animals have innate predispositions to accept some (sweet tasting) and reject other (bitter tasting) foods, and also they acquire feeding responses on the basis of the orosensory properties and postingestive consequences of foods. Animals learn to prefer the flavor of foods and fluids that are associated with positive postingestive nutritional consequences. On the other hand, if animals consume an unfamiliar food or fluid and experience visceral discomfort or malaise, they easily learn to avoid the flavor at subsequent exposures. First, brain regions related to processing of taste information is briefly summarized.

\section{CENTRAL PATHWAYS OF TASTE INFORMATION}

Central gustatory pathways have been well studied in monkeys and rodents especially in rats. Figure 1 shows a schematic diagram of some of the gustatory and related pathways in the rat. Branches of the facial (chorda tympani and greater superficial petrosal), glossopharyngeal, and vagus (superior laryngeal) nerves, which synapse with receptor cells in the taste buds, convey taste messages to the first relay nucleus, the rostral part of the nucleus of the tractus solitarius (NTS). The second relay nucleus for ascending taste inputs is the parabrachial nucleus (PBN) of the pons. The third relay station is the parvocellular part of the ventralis posteromedial thalamic nucleus (VPMpc). This thalamic nucleus sends taste information to the insular cortex (IC). In monkeys, however, ascending fibers of neurons in the gustatory area of the NTS directly reach the VPMpc, bypassing the PBN (Beckstead et al., 1980).

The neural pathway of the brain reward system has also been studied (Wise, 2002). As shown in Figure 1, the essential components are the ventral tegmental area (VTA) of the midbrain which is the origin of the mesolimbic dopamine system, the nucleus accumbens (NAcb) of the ventral forebrain which is an essential interface from motivation (e.g., palatability) to action (e.g., eating), and the ventral palladum (VP) situated between the NAcb and lateral hypothalamus known as the feeding center.

It is not fully understood how the taste system interacts with the reward and feeding system. The amygdala including the central nucleus (CeA) and basolateral nucleus (BLA), the prefrontal cortex (PFC) including the ventrolateral (or anterior sulcal) and dorsomedial cortices and the IC are the candidates for the interfaces between the two systems. The IC sends axons to the PFC (Shi and Cassell, 1998), and the dorsomedial PFC neurons actually respond to gustatory stimuli (Karadi et al., 2005). Among other structures, the PFC is interconnected with the feeding-related subcortical areas such as the VTA (Kosobud et al., 1994) and NAcb (Brog et al., 1993). Behavioral studies have shown that the PFC is associated with various mechanisms in the central feeding control, including conditioned taste aversion (CTA; Hernadi et al., 2000; Karadi et al., 2005). 


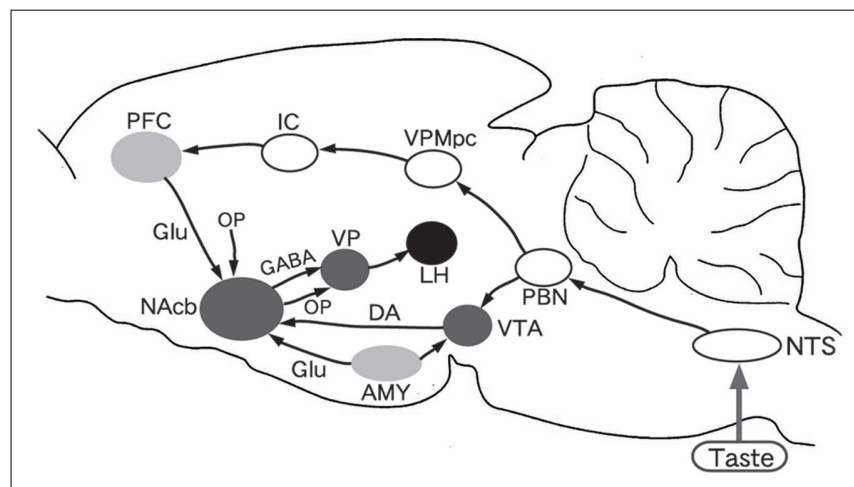

FIGURE 1 | Diagram of connections from the taste system to reward system and feeding center in the rat brain. NTS, nucleus of the tractus solitarius; PBN, parabrachial nucleus; VPMpc, parvocellular part of the ventralis posteromedial thalamic nucleus; IC, insular cortex; PFC, prefrontal cortex; AMY, amygdala; VTA, ventral tegmental area; NAcb, nucleus of accumbens; VP, ventral pallidum; LH, lateral hypothalamic area. DA, dopamine; GABA, $\gamma$-aminobutyric acid; Glu, glutamic acid; OP, opioids.

\section{FLAVOR PREFERENCE}

There are at least two types of learning-based flavor preference: attenuation of neophobia and conditioned flavor preference which consists of conditioned odor preference and conditioned taste preference. When an animal ingests a harmless new substance or liquid, it shows neophobia, or a small intake with caution toward the novel food, and it increases the consumption at subsequent exposures after learning that the food is safe to consume (Bures et al., 1998). Through this process of the attenuation of neophobia (or learned safety), food can be classified as familiar and safe. The IC is suggested to be important in recognition whether the taste is familiar or novel (Gutiérrez et al., 2003; Bahar et al., 2004b). More recent studies suggested that attenuation of neophobia depends not only on the IC (Rodriguez-Ortiz et al., 2005), but also hippocampus and perirhinal cortex (De la Cruz et al., 2008).

When ingestion of novel food (even if it is neutral or mildly aversive) is associated with preferable oral sensations or positive postingestive consequences, the food becomes hedonically positive and preferred on the basis of its taste and odor as cue signals (Sclafani, 2001). Several pairings are required for this effect in contrast to one paring for the aversion learning. Although this phenomenon is collectively called conditioned flavor preference, if you focus on either taste or odor as a cue signal, this learning is referred to as conditioned taste preference and conditioned odor preference, respectively.

Conditioned odor preference can be established when an odor is associated with highly palatable taste (Sakai and Yamamoto, 2001; Sclafani et al., 2001) as well as it is associated with positive postingestive effects (Sclafani and Nissenbaum, 1988). Our ongoing study (Ueji and Yamamoto, 2011) is testing whether weanling (3-week-old) rats can acquire conditioned odor preference, the results being compared with those obtained in young adult (8-week-old) rats. During the acquisition phase of learning, one of the following solutions was presented to each rat for 15 min daily across six consecutive days. Half of the rats in each age group received an unsweetened grape-flavored solution on odd-numbered days and a sweetened (with 2, 20, or 30\% sucrose) cherry-flavored solution on even-numbered days. The remaining rats received a sweetened grape-flavored solution on odd-numbered days and an unsweetened cherry-flavored solution on even-numbered days. In the following test session, each rat received the preference test with unsweetened cherry- and unsweetened grape-flavored solutions simultaneously for $15 \mathrm{~min}$ daily across four consecutive days. Both 3-week-old and 8-weekold rats showed a significant preference for the odor (cherry or grape) previously experienced with $2 \%$ sucrose compared with that previously presented in water. When the concentration of sucrose is increased from 2 to $30 \%$, the 3-week-old rats showed a significant aversion for the odor associated with $30 \%$ sucrose (Figure 2), while 8-week-old rats again showed preference for the odor associated with this strong sucrose, suggesting a hedonic shift from positive to negative with increasing the concentration of sucrose in weanling rats. Our study suggests that aversion to high concentration of sucrose is due to its oral sensation (e.g., osmotic effects) rather than its postingestive caloric effects. Interestingly, both preference and aversion learning acquired at the age of 3 weeks was preserved when retested at the age of 20 weeks (Figure 2). These results suggest that weanling experience of food strongly affects the feeding behavior in adulthood.

Concerning the brain regions responsible for this type of tasteassociated odor preference, Sakai and Yamamoto (2001) demonstrated that rats with lesions in the amygdala showed rapid extinction of preference to the saccharin-associated odor. However, rats with lesions in the IC showed retention of learning similar to that of the control rats. Rats with lesions in the sulcal prefrontal or cingulated cortices showed an intermediate disruptive effect on preference to the saccharin-associated odor. The results also suggested that the odor was associated with the hedonic aspect of taste. Although their results suggested the importance of the

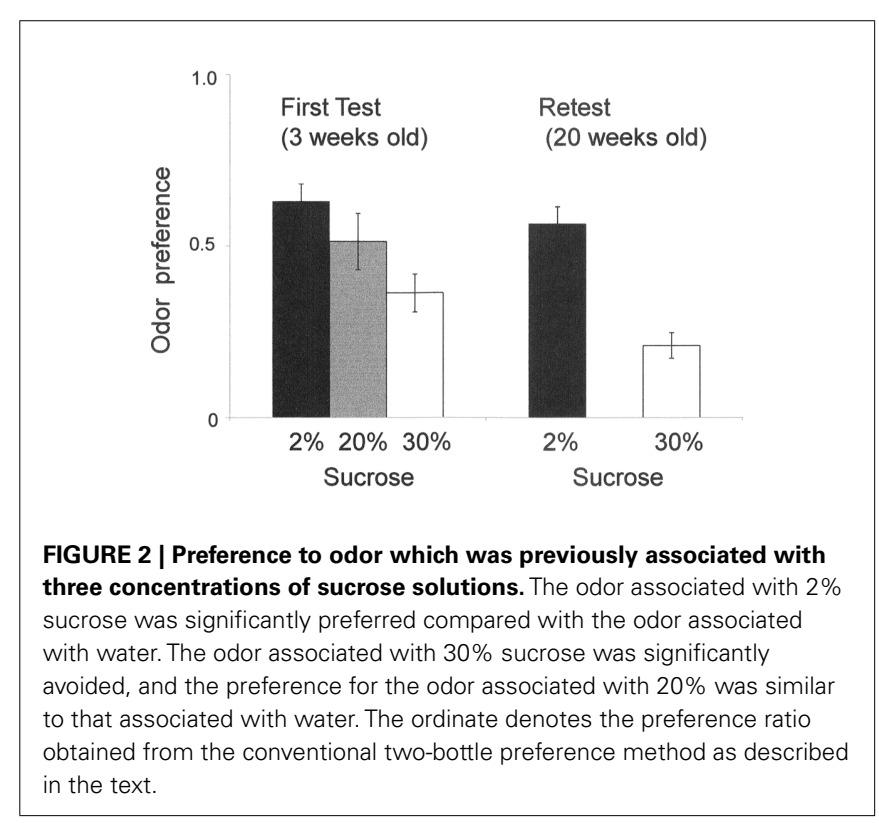


amygdala especially in the retention process of the odor learning, the results also showed that the amygdala-lesioned rats could acquire this learning, which means that the acquisition phase of this learning may involve different parts of the brain in parallel, with either being able to create this type of association learning.

More recently, Desgranges et al. (2010), using a sucrose conditioned odor preference as a flavor experience in rats, demonstrated that the neuronal population activated by both odor and taste strongly increased in the BLA, but not in the IC by using the compartmental analysis of temporal activity with fluorescence in situ hybridization (catFISH) for Arc mRNA. Their results suggest that this greater odor-taste convergence in the BLA is based on the recruitment of a new population of previously silent neural units that acquired the ability to respond to both chemosensory inputs after repeated odor-taste association.

Conditioned taste preference is established when the taste of food is associated with positive postingestive consequences. A representative procedure for this learning is seen in an article by Touzani and Sclafani (2007): rats were trained with distinctive taste stimuli (conditioned stimulus, CS) paired with intragastric infusion of maltodextrin (16\%; unconditioned stimulus, US). The CS solutions contained $0.03 \%$ sucrose octaacetate $+0.2 \%$ saccharin (bitter-sweet) and $2 \% \mathrm{NaCl}+0.2 \%$ saccharin (salty-sweet). It is common to use mildly aversive taste for this type of learning. Although the central neural mechanism of association of taste with postingestive reward is not fully understood, lesion studies suggested that the PBN (Sclafani et al., 2001) and LH (Touzani and Sclafani, 2001) play important roles in conditioned taste preference. Although the amygdala is essential for preference learning when the primary cue is a flavor (both gustatory and olfactory components), it is not critical in taste preference learning (Touzani and Sclafani, 2005). The IC is also not essential for conditioned taste preference and conditioned odor preference (Touzani and Sclafani, 2007).

\section{FLAVOR AVERSION}

In contrast to the flavor preference, when ingestion of a novel food, even if it has preferable taste or odor, is associated with unfavorable postingestive effects or malaise, the food becomes hedonically negative and is avoided and elicits aversive behavior on basis of its taste and odor. This flavor aversion can be divided into conditioned odor aversion and conditioned taste aversion.

Conditioned odor aversion can be acquired in experimental animals by pairing drinking of water with an odor and an intraperitoneal injection of malaise-inducing $\mathrm{LiCl}$ (e.g., Inui et al., 2006). However, animals acquire little aversion to an odor CS, when delivered close to the liquid, with a long CS-US delay, a condition in which aversion to a taste CS can occur (Inui et al., 2006). Animals, however, can acquire strong aversions to the odor CS paired with delayed malaise when it is presented with a taste stimulus as a combined stimulus as the CS. This phenomenon is referred to as taste-potentiated odor aversion (TPOA; Rusiniak et al., 1979). Although direct evidence has not been revealed for the brain mechanism of TPOA, lesion-experiments by Inui et al. (2006) suggested the importance of the amygdala in the formation of TPOA: lesions of the amygdala disrupted both odor and taste aversions, whereas lesions of the thalamic taste area or IC disrupted taste aversion but attenuated only odor aversion. Fernandez-Ruiz et al. (1993) and Desgranges et al. (2009) also reported that the lesions of the IC disrupted the acquisition of aversion to a taste CS without affecting the aversion to an odor CS. Desgranges et al. (2008) suggested that the BLA is necessary for acquisition, consolidation, and retrieval of conditioned odor aversion. Taking into account that there exist neurons that receive convergent inputs of taste and odor (Desgranges et al., 2010) and taste and visceral inputs (Barot et al., 2008), we think that there are neurons that have convergent inputs of taste, odor, and visceral information in the BLA.

\section{CONDITIONED TASTE AVERSION}

Conditioned taste aversion is established when the taste of food (CS) is followed by malaise (US). This association learning between the CS and US is quickly established, and animals remember the taste for a long time, and reject its ingestion at subsequent exposures (Garcia et al., 1955; Bures et al., 1998). After the acquisition of CTA to the CS, the taste quality may not change, but the hedonic aspect changes drastically from positive to negative. On the view of a number of previous researches, behavioral and neural characteristics of CTA can be elucidated by the following five items: (1) alertness (novelty of CS), (2) association between CS and US, (3) avoidance, (4) aversion (hedonic shift from positive to negative), and (5) augmentation of responses to the CS (see Figure 3).

\section{ALERTNESS (NOVELTY OF CS)}

It is well documented that strong CTA can be acquired when the CS is novel rather than familiar (Bures et al., 1998). Novelty plays a key role in alerting animals to be cautious toward the food (neophobia). In their investigation of the role of the cholinergic system in the IC, Miranda et al. (2000) found that novel tastes significantly elevated acetylcholine (Ach) levels, whereas familiar tastes did not. Furthermore, inactivation of the nucleus basalis magnocellularis, which is the origin of cholinergic projections to IC, before presentation of a novel taste blocked the increase in Ach release and impaired CTA acquisition. On the basis of these findings, Clark

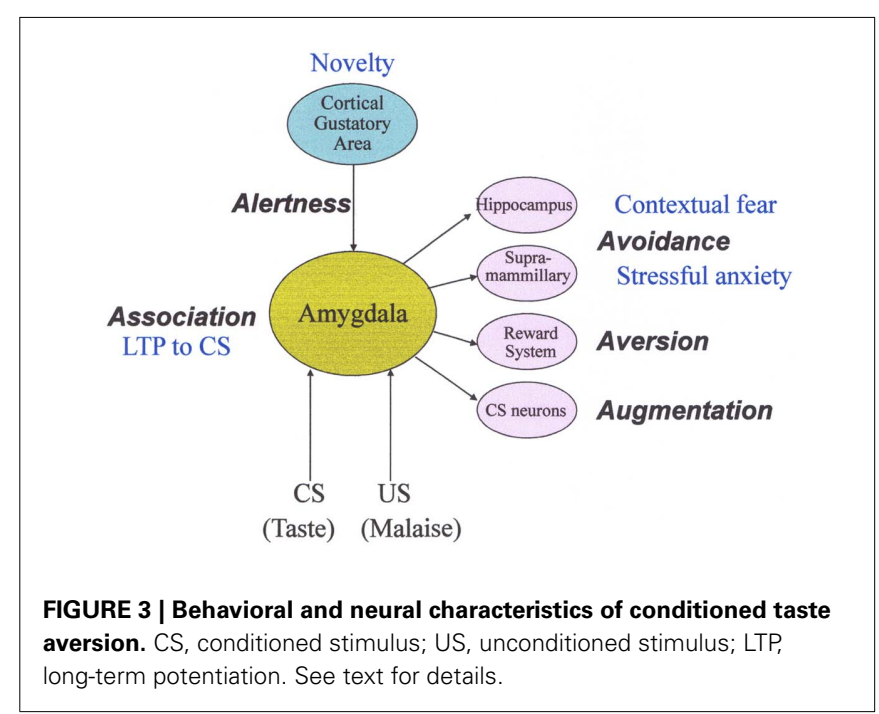


and Bernstein (2009) tried to enhance the salience of a familiar CS (saccharin) by infusing carbachol, a direct cholinergic agonist, before CTA and found that rats were able to acquire CTA to familiar saccharin. They also found that familiar CS associated with illness after carbachol, but not vehicle, induced significant elevation of Fos-like immunoreactivity in the amygdala. These results support the notion that Ach activity in the IC provides a critical signal of taste novelty that facilitates CTA acquisition. Thus, familiarity information is stored in the IC and is sent to the subcortical taste relay stations (Yamamoto et al., 2009).

\section{ASSOCIATION BETWEEN CS AND US}

Long-term potentiation occurs in the BLA in response to a single electrical stimulation of the PBN. When we used fairly a large electrode, both gustatory and general visceral routes were stimulated, and activity of mass neurons was recorded as evoked potentials. After repetitive stimulation of the PBN, the evoked potential to single stimulation of the PBN was potentiated by more than $50 \%$ of the original response (Yamamoto and Yasoshima, 2007). Once a paring of the CS and US occurs, the established long-term potentiation to the CS is the basis of the aversive learning. In fact, Yasoshima et al. (2006) showed by the Fos-like immunoreactivity analysis that sucrose CS induced strong activation of BLA neurons to re-exposure to sucrose after the acquisition of CTA.

Concerning the role of amygdala in CTA, a number of studies have dealt with the functions of the amygdalar subnuclei in the formation of CTA. Although the studies have yielded inconsistent behavioral results, overall electrolytic or excitotoxic lesions show little, if any, involvement of the CeA in CTA (Yamamoto et al., 1995; Morris et al., 1999). Our previous lesion-behavioral studies (Yamamoto et al., 1995) showed that lesions of the CeA had little effects on CTA, and lesions of the BLA severely impaired CTA. Consisting with these findings, a recent study shown below has demonstrated evidence that BLA is a site for CS-US convergence.

Using the catFISH imaging analysis, Barot et al. (2008) provided evidence that, during CTA acquisition, CS and US information converges exclusively on a subset of neurons in the BLA, but not in the IC, when presentation of the stimuli is effective in promoting learning (novel CS-US paring) but not effective when the same stimuli are presented in an ineffective manner (familiar CS-US pairing or backward CS-US pairing). On the basis of their findings, they have proposed a model in which potentiation of US responses by "novel" CS presentation is key to coincidental activation and its sensitivity to "temporal order" (CS-US pairing but not US-CS paring). The existence of neurons receiving convergence of information from pathways mediating CS and US and showing strong and prolonged activation is the basis of association memory formation and is critical for subsequent plasticity.

\section{AVOIDANCE}

Yasoshima et al. $(2005,2007)$ found that the supramammillary nucleus and thalamic paraventricular nucleus were activated by retrieval of the CS after the acquisition of CTA in the overall survey of the brain with the Fos-like immunoreactivity technique. These two regions are suggested to be involved in the expression of anxiety and psychological stress (Wirtshafter et al.,
1998; Bubser and Deutch, 1999), and Yasoshima et al. (2005) have suggested that the supramammillary nucleus is activated by memory-elicited discomfort during retrieval of CTA.

Since lesions of hippocampus induce essentially no effect on the acquisition itself of CTA (Yamamoto et al., 1995), CTA is generally accepted as "non-hippocampal" learning, Considering the well-documented facts that the hippocampus is concerned with context fear learning, the hippocampus may modulate CTA in some respects. In line with this notion and on the basis of their previous finding that environmental and temporal contexts can modulate taste aversion learning (Moron et al., 2002), Gallo and her colleagues (Manrique et al., 2009a,b) have studied modulation of taste aversion learning by the time of day (morning 9:00 or evening 19:00) in rats of different ages ranging from 32-day-old to 25-month-old and the role of hippocampus in such a modulation. Their results suggest that the ability to form segregated representations of a complex experience is impaired in aging and abolished by lesions of the dorsal hippocampus.

\section{AVERSION (HEDONIC SHIFT FROM POSITIVE TO NEGATIVE)}

Yasoshima et al. (2006) also found that the BLA, extended amygdala and NAcb were also activated during the retrieval phase of CTA. The latter two regions belong to the reward system. CS information from the BLA reaches the NAcb directly or via the extended amygdala (Groenewegen et al., 1999; Shammah-Lagnado et al., 1999, 2001). The $r$-aminobutyric acidergic (GABAergic) neurons in the NAcb send axons to the VP as the main output target (Zahm et al., 1985). The CS induced strong activity in the BLA where little activity was induced by the CS in control animals, suggesting a key role of the BLA in the formation of CTA.

The reward system may be involved in aversive reactions to the CS after the acquisition of CTA. To elucidate the role of the VP in the expression of CTA, Inui et al. (2007) examined the effects of microinjections of a $\mathrm{GABA}_{\mathrm{A}}$ receptor antagonist, bicuculline, on the intake of CS in a retrieval test. They showed that the blockade of $\mathrm{GABA}_{\mathrm{A}}$ receptors in the VP by microinjections of bicuculline disrupted the expression of CTA (Figure 4B), and have suggested that this is due to elimination of aversive responses to the CS. This finding suggests that the GABAergic neurotransmission in the VP is involved in expression of aversive responses to CS, we actually confirmed the increase of the level of extracellular GABA release in the VP using microdialysis technique (Figure 4A; Inui et al., 2009). Using a newly developed manganese-enhanced MRI technique, Inui et al. (2011) actually demonstrated an activated pattern in projective neurons from the NAcb to VP by the presentation of a learned aversive taste stimulus inducing rejective behaviors in the retrieval of CTA. We conclude from these findings that the CS presentation after acquisition of CTA increases the extracellular GABA release in the VP through the activation of the NAcb receiving inputs from the amygdala, inducing the expression of aversive responses to the CS and the inhibition of consumption of the CS.

The suggestion that the increase of GABA level in the VP induces expression of aversive responses is based on our previous finding (Shimura et al., 2006). Microinjections of muscimol, a $\mathrm{GABA}_{\mathrm{A}}$ receptor agonist, significantly decreased the consumption of water, saccharin, and quinine solutions in rats (Figure 4C). Interestingly, the rats showed strong aversive taste reactivity, such 

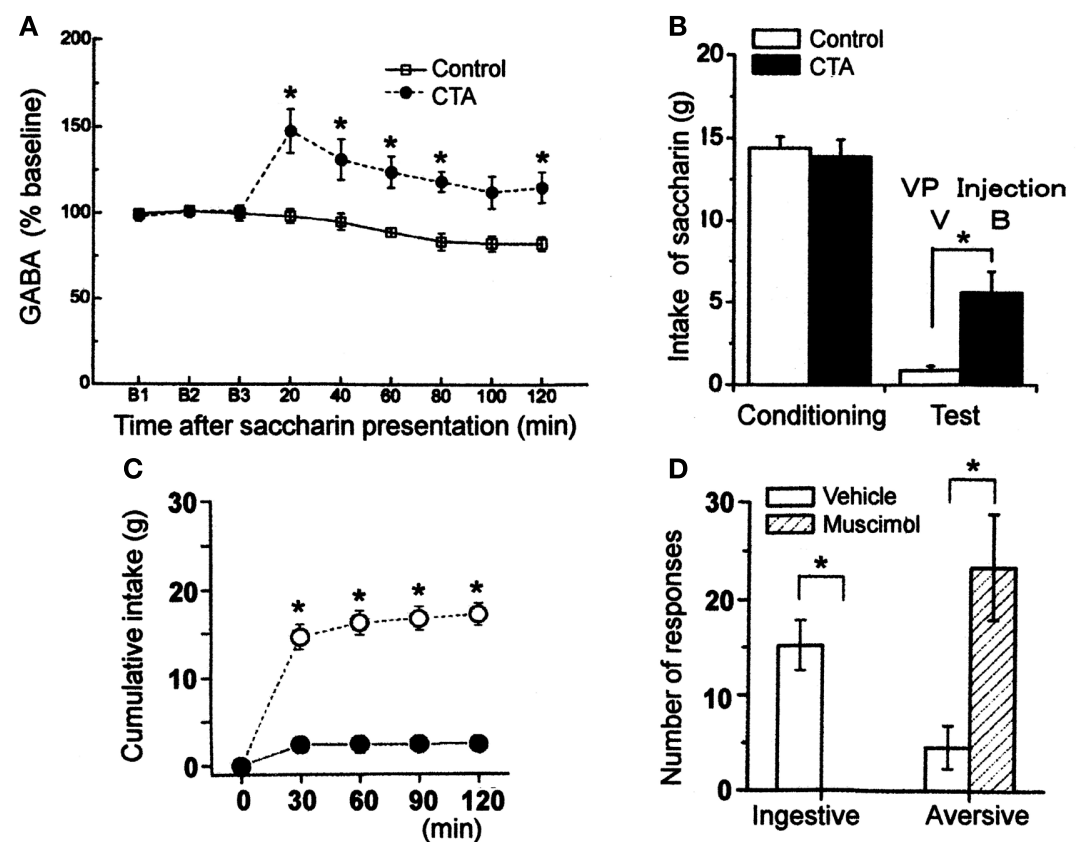

FIGURE 4 | Functional importance of GABA in the ventral pallidum (VP). (A) Intraoral infusion of saccharin solution after CTA acquisition increased GABA release in the VP. (B) The intake of saccharin in the vehicle-injected control group (V) was very small after CTA acquisition, but it increased significantly after microinjection of bicuculline [50 ng (B)]. (C) Muscimol (100 ng) injected into the VP

as chin rubbing, gaping, forelimb flailing, and head shaking, and decreased ingestive reactivity, such as tongue protrusions and rhythmic mouth movements, after the voluntary intake of fluids or the intraoral infusion of normally preferred water or saccharin solution (Figure 4D). Thus, the VP is suggested to participate in aversive aspects of ingestive behavior through robust GABAergic neurotransmission. Increased GABAergic transmission in the VP might activate various brain sites responsible for the aversive taste reactivity, including the parvicellular subdivision of the intermediate nucleus of the NTS (iNTSpc), a region strongly activated in association with CTA expression (Schafe et al., 1995). The iNTSpc, which is proved to receive direct projection from the amygdala (Spray and Bernstein, 2004), might receive indirect inputs from the VP to exert aversive reactions by exposure to a conditioned aversive taste.

\section{AUGMENTATION}

Perceived intensity of the CS becomes stronger after the acquisition of CTA. Shimura et al. (1997) recorded neuronal responses to taste stimuli from the PBN of anesthetized rats. Animals were separated into two groups: the CTA group that had acquired a taste aversion to $0.1 \mathrm{M} \mathrm{NaCl}(\mathrm{CS})$ by paired presentation of an i.p. injection of $\mathrm{LiCl}$ (US), and the control group without CTA experience. Taste-responsive neurons in the CTA group showed larger responses to $\mathrm{NaCl}$ than in the control group. Tokita et al. (2004, 2007) found that the enhanced responses to the CS were observed exclusively in amiloride-sensitive $\mathrm{NaCl}$-best neurons, but suppressed the intake of saccharin which was significantly different from the intake after vehicle injection at each time point. (D) Mean \pm SEM number of ingestive and aversive taste reactivity responses to intraoral infusion of $5 \mathrm{mM}$ saccharin after microinjection of muscimol or vehicle in the VP. ${ }^{*} p<0.05$. (Modified from Shimura et al., 2006; Inui et al., 2007, 2009.)

neither in amiloride-insensitive $\mathrm{NaCl}$-best nor any other best neurons. They have suggested that amiloride-sensitive components of $\mathrm{NaCl}$-best neurons play a critical role in the recognition of the distinctive taste of $\mathrm{NaCl}$. Not only PBN neurons, but CGA neurons (Yamamoto et al., 1989; Yasoshima and Yamamoto, 1998) and amygdalar neurons (Yamamoto and Fujimoto, 1991; Yasoshima et al., 1995) exhibit enhanced responses to the CS after CTA acquisition. Augmentation of CS responses enables the animal to facilitate detecting and avoiding the harmful substance.

The aversive memory is stored in the IC, amygdala, and others in the long term after consolidation process accompanying protein synthesis which is derived from augmented activation of relevant neurons (e.g., Shema et al., 2007, 2011). Consolidated memory regains the labile state when retrieved, which is known as reconsolidation. Extinction, a decline in the frequency or intensity of the conditioned response following the withdrawal of reinforcement, is not loss of the original memory, but is a new learning accompanying consolidation and reconsolidation. Consolidation, reconsolidation, and extinction have been studied in taste aversion learning (e.g., Berman and Dudai, 2001; Koh and Bernstein, 2003; Bahar et al., 2004a; Garcia-delaTorre et al., 2010). These are important issues to be more clarified in the future.

\section{ACKNOWLEDGMENTS}

This article was supported by Grant-in-Aid for Scientific Research from the Japan Society for the Promotion of Science (21500808 to Takashi Yamamoto and 23500986 to Kayoko Ueji). 


\section{REFERENCES}

Bahar, A., Dorfman, N., and Dudai, Y. (2004a). Amygdalar circuits required for either consolidation or extinction of taste aversion memory are not required for reconsolidation. Eur. J. Neurosci. 19, 1115-1118.

Bahar, A., Dudai, Y., and Ahissar, E. (2004b). Neural signature of taste familiarity in the gustatory cortex of the freely behaving rat. J. Neurophysiol. 92, 3298-3308.

Barot, S. K., Kyono, Y., Clark, E. W., and Bernstein, I. L. (2008). Visualizing stimulus convergence in amygdala neurons during associative learning. Proc. Natl. Acad. Sci. U.S.A. 105, 20959-20963.

Beckstead, R. M., Morse, J. R., and Norgren, R. (1980). The nucleus of the solitary tract in the monkey: projections to the thalamus and brain stem nuclei. J. Comp. Neurol. 190, 259-282.

Berman, D. E., and Dudai, Y. (2001). Memory extinction, learning anew, and learning the new: dissociations in the molecular machinery of learning in cortex. Science 291, 2417-2419.

Brog, J. S., Salyapongse, A., Deutch, A. Y., and Zahm, D. S. (1993). The patterns of afferent innervation of the core and shell in the "accumbens" part of the rat ventral striatum: immunohistochemical detection of retrogradely transported fluoro-gold. J. Comp. Neurol. 338, 255-278.

Bubser, M., and Deutch, A. Y. (1999). Stress induces Fos expression in neurons of the thalamic paraventricular nucleus that innervate limbic forebrain sites. Synapse 32, 13-22.

Bures, J., Bermudez-Rattoni, F., and Yamamoto, T. (1998). Conditioned Taste Aversion: Memory of a Special Kind. New York: Oxford University Press.

Clark, E. W., and Bernstein, I. L. (2009). Boosting cholinergic activity in gustatory cortex enhances the salience of a familiar conditioned stimulus in taste aversion learning. Behav. Neurosci. 123, 764-771.

De la Cruz, V., Rodriguez-Ortiz, C. J., Balderas, I., and Bermudez-Rattoni, F. (2008). Medial temporal lobe structures participate differentially in consolidation of safe and aversive taste memories. Eur. J. Neurosci. 28, 1377-1381.

Desgranges, B., Levy, F., and Ferreira, G. (2008). Anisomycin infusion in amygdala impairs consolidation of odor aversion memory. Brain Res. 1236, 166-175.

Desgranges, B., Ramirez-Amaya, V., Ricano-Cornejo, I., Levy, F., and Ferreira, G. (2010). Flavor preference learning increases olfactory and gustatory convergence onto single neurons in the basolateral amygdala but not in the insular cortex. PLoS ONE 5, e10097. doi: 10.1371/journal.pone.0010097

Desgranges, B., Sevelinges, Y., Bonnefond, M., Levy, F., Ravel, N., and Ferreira, G. (2009). Cortical role of insular cortex in taste but not odour aversion memory. Eur. J. Neurosci. 29, 1654-1662.

Fernandez-Ruiz, J., Miranda, M. I. Bermudez-Rattoni, F., and DruckerColin, R. (1993). Effects of catecholaminergic depletion of the amygdala and insular cortex on the potentiation of odor by taste aversions. Behav. Neural Biol. 60, 189-191.

Garcia, J., Kimeldorf, D. J., and Koelling, R. A. (1955). Conditioned aversion to saccharin resulting from exposure to gamma radiation. Science 122, 157-158.

Garcia-delaTorre, P., Rodriguez-Ortiz, C. J., Balderas, I., and BermudezRattoni, F. (2010). Differential participation of temporal structures in the consolidation and reconsolidation of taste aversion extinction. Eur. J. Neurosci. 32, 1018-1023.

Groenewegen, H. J., Wright, C. I., Beijer, A. V., and Voorn, P. (1999). Convergence and segregation of ventral striatal inputs and outputs. Ann. N. Y. Acad. Sci. 877, 49-63.

Gutiérrez, R., Téllez, L. A., and Bermúdez-Rattoni, F. (2003). Blockade of cortical muscarinic but not NMDA receptors prevents a nove taste from becoming familiar. Eur. J. Neurosci. 17, 1556-1562.

Hernadi, I., Karadi, Z., Vígh, J., Petyko, Z., Egyed, R., Berta, B., and Lenard, L. (2000). Alterations of conditioned taste aversion after microiontophoretically applied neurotoxins in the medial prefrontal cortex of the rat. Brain Res. Bull. 53, 751-758.

Inui, T., Inui-Yamamoto, C., Yoshioka, Y., Ohzawa, I., and Shimura, T. (2011). Activation of projective neurons from the nucleus accumbens to ventral pallidum by a learned aversive taste stimulus in rats: a manganese-enhanced magnetic resonance imaging study. Neuroscience 177, 66-73.

Inui, T., Shimura, T., and Yamamoto, T. (2006). Effects of brain lesions on taste-potentiated odor aversion in rats. Behav. Neurosci. 120, 590-599.

Inui, T., Shimura, T., and Yamamoto T. (2007). The role of the ventral pallidum GABAergic system in conditioned taste aversion: effects of microinjections of a GABAA receptor antagonist on taste palatability of a conditioned stimulus. Brain Res 1164, 117-124.

Inui, T., Yamamoto, T., and Shimura T. (2009). The GABAergic transmission in the rat ventral pallidum mediates a palatability shift in conditioned taste aversion. Eur. J. Neurosci. 30, 110-115.

Karadi, Z., Lukats, B., Papp, Sz. Egyed, R., Lenard, L., and Takacs, G. (2005). Involvement of forebrain glucose-monitoring neurons in taste information processing: electrophysiological and behavioral studies. Chem. Senses 30, i168-i169.

Koh, M. T., and Bernstein, I. L. (2003). Inhibition of protein kinase A activity during conditioned taste aversion retrieval: interference with extinction or reconsolidation of a memory? Neuroreport 14, 405-407.

Kosobud, A. E., Harris, G. C., and Chapin, J. K. (1994). Behavioral associations of neuronal activity in the ventral tegmental area of the rat. J. Neurosci. 14, 7117-7129.

Manrique, T., Gamiz, F., Moron, I. Ballesteros, M. A., and Gallo, M. (2009a). Peculiar modulation of taste aversion learning by the time of day in developing rats. Dev. Psychobiol. 51, 147-157.

Manrique, T., Moron, I., Ballesteros, M. A., Guerrero, R. M., Fenton, A. A. and Gallo, M. (2009b). Hippocampus, aging, and segregating memories. Hippocampus 19, 57-65.

Miranda, M. I., Ramirez-Lugo, L., and Bermudez-Rattoni, F. (2000). Cortical cholinergic activity is related to the novelty of the stimulus. Brain Res. 882, 230-235.

Moron, I., Manrique, T., Molero, A. Ballesteros, M. A., Gallo, M., and Fenton, A. (2002). The contextual modulation of conditioned taste aversions by the physical environment and time of day is similar. Learn. Mem. 9, 218-223.

Morris, R., Frey, S., Kasambira, T., and Petrides, M. (1999). Ibotenic acid lesions of the basolateral, but not the central, amygdala interfere with conditioned taste aversion: evidence from a combined behavioral and anatomical tract-tracing investigation. Behav. Neurosci. 113, 291-302.

Rodriguez-Ortiz, C. J., De la Cruz, V., Gutierrez, R., and BermudezRattoni, F. (2005). Protein synthesis underlies post-retrieval memory consolidation to a restricted degree only when updated information is obtained. Learn. Mem. 12, 533-537.

Rusiniak, K. W., Hankins, W. G., Garcia, J., and Brett, L. P. (1979). Flavorillness aversions: potentiation of odor by taste in rats. Behav. Neural Biol. 25, 1-17.
Sakai, N., and Yamamoto, T. (2001). Effects of excitotoxic brain lesions on taste-mediated odor learning in the rat. Neurobiol. Learn. Mem. 75 , 128-139.

Schafe, G. E., Seeley, R. J., and Bernstein, I. (1995). Forebrain contribution to the induction of a cellular correlate of conditioned taste aversion in the nucleus of the solitary tract. $J$. Neurosci. 15, 6789-6796.

Sclafani, A. (2001). Postingestive positive controls of ingestive behavior. Appetite 36, 79-83.

Sclafani, A., Azzara, A. V., Touzani, K., and Grigson, P. S. Norgren, R. (2001). Parabrachial nucleus lesions block taste and attenuate flavor preference and aversion conditioning in rats. Behav. Neurosci. 115, 920-933.

Sclafani, A., and Nissenbaum, J. W. (1988). Robust conditioned flavor preference produced by intragastric starch infusions in rats. Am. J. Physiol. 255, R672-R675.

Shammah-Lagnado, S. J., Alheid, G. F., and Heimer, L. (1999). Afferent connections of the interstitial nucleus of the posterior limb of the anterior commissure and adjacent amygdalostriatal transition area in the rat. Neuroscience 94 1097-1123.

Shammah-Lagnado, S. J., Alheid, G. F., and Heimer, L. (2001). Striatal and central extended amygdala parts of the interstitial nucleus of the posterior limb of the anterior commissure: evidence from tract-tracing techniques in the rat. J. Comp. Neurol. 439, 104-126.

Shema, R., Haramati, S., Ron, S., Hazvi, S., Chen, A., Sacktor, T. C., and Dudai, Y. (2011). Enhancement of consolidated long-term memory by overexpression of protein kinase $\mathrm{M \zeta}$ in the neocortex. Science 331, 1207-1210

Shema, R., Sacktor, T. C., and Dudai, Y. (2007). Rapid erasure of long-term memory associations in the cortex by an inhibitor of $\mathrm{PKM} \zeta$. Science 317, 951-953.

Shi, C-J., and Cassell, M. D. (1998). Cortical, thalamic, and amygdaloid connections of the anterior and posterior insular cortices. J. Comp. Neurol. 399, 440-468.

Shimura, T., Imaoka, H., and Yamamoto, T. (2006). Neurochemical modulation of ingestive behavior in the ventral pallidum. Eur. J. Neurosci. 23, 1596-1604.

Shimura, T., Tanaka, H., and Yamamoto, T. (1997). Salient responsiveness of parabrachial neurons to the conditioned stimulus after the acquisition of taste aversion learning in rats. Neuroscience 81, 239-247. 
Spray, K. J., and Bernstein, I. (2004). Afferent and efferent connections of the parvicellular subdivision of iNTS: defining a circuit involved in taste aversion learning. Behav. Brain Res. 154, 85-97.

Tokita, K., Karadi, Z., Shimura, T., and Yamamoto, T. (2004). Centrifugal inputs modulate taste aversion learning associated parabrachial neuronal activities. J. Neurophysiol. 92, 265-279.

Tokita, K., Shimura, T., Nakamura, S., Inoue, $\mathrm{T}$, and Yamamoto, $\mathrm{T}$. (2007). Involvement of forebrain in parabrachial neuronal activation induced by aversively conditioned taste stimuli in the rat. Brain Res. 1141, 188-196.

Touzani, K., and Sclafani, A. (2001). Conditioned flavor preference and aversion: role of the lateral hypothalamus. Behav. Neurosci. 115, 84-93.

Touzani, K., and Sclafani, A. (2005). Critical role of amygdala in flavor but not taste preference learning in rats. Eur. J. Neurosci. 22, 1767-1774.

Touzani, K., and Sclafani, A. (2007). Insular cortex lesions fail to block flavor and taste preference learning in rats. Eur. J. Neurosci. 26, 1692-1700.

Ueji, K., and Yamamoto, T. (2011). Conditioned flavor preference in weanling and adult rats. Chem. Senses 36, J8.

Wirtshafter, D., Stratford, T. R., and Shim, I. (1998). Placement in a novel environment induces Fos-like immunoreactivity in supramammillary cells projecting to the hippocampus and midbrain. Brain Res. 789, 331-334.

Wise, R. A. (2002). Brain reward circuitry: insights from unsensed incentives. Neuron 36, 229-240.

Yamamoto, T., and Fujimoto, Y. (1991). Brain mechanisms of taste aversion learning in the rat. Brain Res. Bull. 27, 403-406.

Yamamoto, T., Fujimoto, Y., Shimura, T., and Sakai, N. (1995). Conditioned taste aversion in rats with excitotoxic brain lesions. Neurosci. Res. 22, 31-49.

Yamamoto, T., Matsuo, R., Kiyomitsu, Y., and Kitamura, R. (1989). Taste responses of cortical neurons in freely ingesting rats. J. Neurophysiol. 61, 1244-1258.

Yamamoto, T., Takemura, M., Inui, T., Torii, K., Maeda, N., Ohmoto M., Matsumoto, I., and Abe, K. (2009). Functional organization of the rodent parabrachial nucleus. Ann. N. Y. Acad. Sci. 1170, 378-382.
Yamamoto, T., and Yasoshima, Y. (2007) "Electrophysiological representation of taste memory," in Neural Plasticity and Memory: From Genes to Brain Imaging, ed. F. BermudezRattoni (Boca Raton: CRC Press), 113-128.

Yasoshima, Y., Scott, T. R., and Yamamoto, T. (2005). Involvement of the supramammillary nucleus in aversive conditioning. Behav. Neurosci. 119, 1290-1297.

Yasoshima, Y., Scott, T. R., and Yamamoto, T. (2006). Memorydependent c-Fos expression in the nucleus accumbens and extended amygdala following the expression of a conditioned taste aversive in the rat. Neuroscience 141, 35-45.

Yasoshima, Y., Scott, T. R., and Yamamoto, T. (2007). Differential activation of anterior and midline thalamic nuclei following retrieval of aversively motivated learning tasks. Neuroscience 146, 922-930.

Yasoshima, Y., Shimura, T., and Yamamoto, T. (1995). Single unit responses of the amygdala after conditioned taste aversion in conscious rats. Neuroreport 6, 2424-2428.

Yasoshima, Y., and Yamamoto, T. (1998). Short-term and longterm excitability changes of the insular cortical neurons after the acquisition of taste aversion learning in behaving rats. Neuroscience 284, 1-5.

Zahm, D. S., Zaborszky, L., Alones, V. E., and Heimer, L. (1985). Evidence for the coexistence of glutamate decarboxylase and Met-enkephalin immunoreactivities in axon terminals of rat ventral pallidum. Brain Res. 325, 317-321.

Conflict of Interest Statement: The authors declare that the research was conducted in the absence of any commercial or financial relationships that could be construed as a potential conflict of interest.

Received: 26 June 2011; accepted: 15 August 2011; published online: 05 September 2011.

Citation: Yamamoto $T$ and $U e j i ~ K$ (2011) Brain mechanisms of flavor learning. Front. Syst. Neurosci. 5:76. doi: 10.3389/fnsys.2011.00076

Copyright (๑) 2011 Yamamoto and Ueji. This is an open-access article subject to a non-exclusive license between the authors and Frontiers Media SA, which permits use, distribution and reproduction in other forums, provided the original authors and source are credited and other Frontiers conditions are complied with 